\title{
Lightness matching for different visual routes through a compound scene'
}

HOWARD R. FLOCK, ALAN WILSON AND SONJA POIZNER

YORK UNIVERSITY

Left and right halves of a visual display were covered with inducing fields (IFs) of different lightnesses. S's monocular gaze moved over an irreversible route from a neutral Munsell target to a CO series through either the left- or right-side IFs. For the 16 Ss there were 8 different IFs, varying from light to dark. For each of three different gray targets Munsell CO choices varied directly with the lightness of the IFs through which the gaze was routed rather than with the lightness of the total presented display. A replication with modifications is also reported.

Theorists of brightness contrast have insisted that attempts to predict the appearance of an achromatic color (AC) must take account of the preceding stimulus variables as well as of the focal and surrounding variables (Helson, 1964; Jameson \& Hurvich, 1959; Judd, 1941). Nevertheless, the empirical literature on AC perception has been preoccupied with simultaneous rather than with successive contrast, unless one construes the work on negative after-images, adaptationlevel, time errors, and even figural after-effects and metacontrast as being synonomous with successive contrast (SC).

Judd (1941) proposed that the same principles which held for simultaneous contrast would apply to SC. The recent literature on simultaneous contrast has demonstrated that high-luminance surrounds darken lowluminance infields, whereas low-luminance surrounds have little or no effect on high-luminance infields (Diamond, 1953; Heinemann, 1955; Leibowitz, Mote, \& Thurlow, 1953). If similar principles held for sequential inspection of different achromatic fields, then looking at interpolated white inducing fields (IFs) following the inspection of a mid-gray target (ST) should darken an array of Munsell comparison (CO) chips. A S required to choose a CO that matched the ST would presumably select a $\mathrm{CO}$ that would be too light. Correspondingly, as the interpolated IFs were made darker and darker there would be less and less effect on the $\mathrm{CO}$ chips and the CO choices would be correspondingly darker.

In the experiment described below three different STs were used (Munsell 3.5, 5.5, and 7.5). Between viewing the ST and viewing the 17 step (1.5 to 9.5) neutral Munsell CO series, IFs were interpolated which varied in lightness over the Munsell range and which varied in spatial (and therefore in temporal) distance from the $\mathrm{CO}$ series. It was expected that the interpolated IFs would act forward on the CO patches in the manner hypothesized above.
Stimuli

Two poster-boards, $44 \mathrm{in}$. high and $64 \mathrm{in}$. wide, were painted a flat N. 5.5 Munsell gray. A CO series of 17 Munsell Chips (from 1.5 to 9.5), each 1 in. by 2 in., was mounted at the top-center of each board (see Figs. 1a and 1b). Randomly different trapezoids corresponding to Munsells 2 to 8 were permanently mounted on one poster board (see Fig. 1a). On the other, the series of gray trapezoids was dark-biased (corresponding to Munsell's 2 to 4.5 ) on the right side and lightbiased (corresponding to Munsell's 6 to 8 ) on the left (see Fig. 1b).

In the left- and right-bottom corners of each display an $8.5 \times 11$ in. Munsell white (9.5) or black (1.5) sheet could be mounted to serve as a first IF. Finally, at the bottom-center of each display a Munsell neutral gray ST target (either 3.5, 5.5, or 7.5), 3 in. wide and 5 in. high, could be mounted (see Figs. 1a and 1b). Between the targets and the nearest IFs was a space of $1.5 \mathrm{in}$. The shortest angular distance from the top of a ST to the nearest edge of a $\mathrm{CO}$ was $18^{\circ}$.
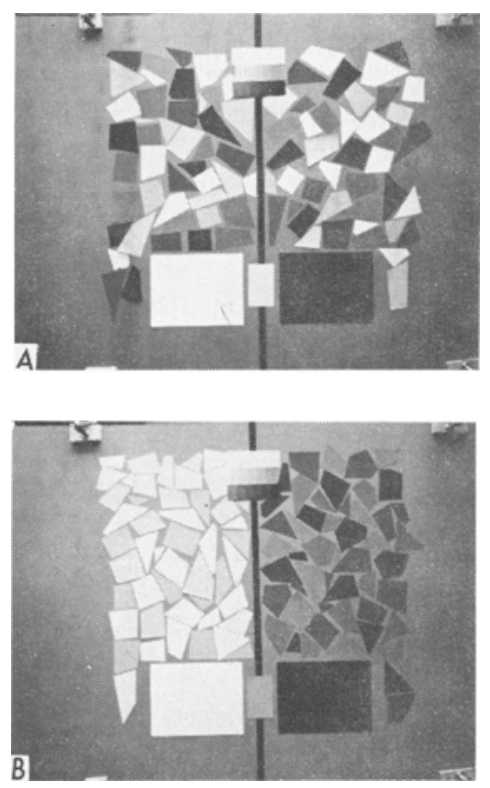

Fig. 1. Distribution, Iocation, and relative lightness of inducingfields, comparison-series, and targets. 
The poster-boards were viewed monocularly (through a $28^{\circ}$ black reduction screen allowing a view of 962 sq. in. of display area) with the eye at a distance of $70.5 \mathrm{in}$. from the center of the display. With these arrangements IFs covered approximately $75 \%$ of the visible area of a display. On the back side of the partition holding the eye-piece, at a radius of $17 \mathrm{in.} \mathrm{from}$ the center of the eye-piece, were mounted four $7.5 \mathrm{in}$. fluorescent tubes, GE F6T5-CW. The luminances of the Munsell 5.5 poster-board at 12, 3,6, and 9 o'clock were $1.0 \mathrm{ft} .-\mathrm{L}$, and at the center, $1.2 \mathrm{ft} .-\mathrm{L}$, as measured from the viewing position with an SEI Exposure Photometer.

The three STs and the two IFs in the bottom-corners were mounted on .002 shim steel and were held in position by strip magnets placed behind the posterboards. With this arrangement they were easily and rapidly interchangeable.

\section{Procedure}

Sixteen students at York University, eight men and eight women, with median age of 21 and with uncorrected $20 / 20$ vision in at least one eye, participated. Of these only three had taken any psychology courses. All Ss wore an eye-patch over their non-preferred eye throughout the experiment.

Each S was asked to look at a ST for approximately 6 sec. He was then directed to shift his gaze either to the left or to the right bottom corner and to look at the first IF for approximately $6 \mathrm{sec}$. The $S$ was then directed to move his eyes in a vertical sweep (lasting approximately $1.5 \mathrm{sec}$.) through the second IF (the vertical array of gray trapezoids lying in the half of the poster-board directly above the first IF) to the COs. He was required to select a $\mathrm{CO}$ that appeared to match the ST. Each $S$ was continuously told not to look back during this procedure. On each trial $E$ verbally guided S's gaze from ST to the first IF, vertically through the second $I F$, to the COs. Between trials $S$ looked away from the eye-piece at a black surface measuring $.005 \mathrm{ft}$. $L$. Intertrial delays were on the average 60 sec.

This procedure yielded eight treatment levels that varied the amount of whiteness interpolated between viewing the ST and COs and varied the distance of the IFs from the COs. These eight conditions involved eye-movements to left (L) or right (R) into a white (W) or black (B) IF and then vertical eye-movements through a second IF composed of randomly-different achromatic gray patches varying from high to low reflectances (M) or composed of high-reflectance patches $(\mathrm{H})$ or of lowreflectance patches (D). Thus, the eight conditions were: LW-H-Left to white IF, then through a second IF of

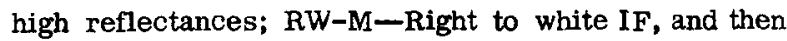
IF of high and low reflectances; LW-M-Left to white, then high and low reflectances; RW-D-Right to white and then low reflectances; LB-M-Left to black, then high and low reflectances; LB-H-Left to black, then
Table 1. Mean ranks for sixteen subjects over eight inducing fields for three standards and mean ranks of the mean ranks

\begin{tabular}{lllllllll} 
ST & LW-H & RW-M & LW-M & RW-D & LB-M & LB-H & RB-M & RB-D \\
\hline 3.5 & 3.62 & 3.97 & 3.88 & 3.88 & 5.00 & 4.50 & 5.19 & 5.97 \\
5.5 & 3.78 & 3.78 & 3.78 & 4.09 & 4.81 & 4.81 & 4.97 & 5.97 \\
7.5 & 3.34 & 3.25 & 4.0 & 4.66 & 4.69 & 5.44 & 4.75 & 5.88 \\
Means* & 1.67 & 2.33 & 2.50 & 3.50 & 5.30 & 5.83 & 6.67 & 8.00 \\
\hline
\end{tabular}

* These ranks were calculated by ranking the mean ranks at each level of the Table.

high reflectances; RB-M-Right to black, then high and low reflectances; RB-D-Right to black, then low reflectances.

The apparent lightness of the three STs was judged under each of these eight conditions by all Ss, a total of 24 judgments for each S. For half the Ss the lightness of the three STs was first judged under conditions RW-M; LW-M; LB-M, and RB-M, followed by the other 12 treatment combinations. The reverse was true for the second half of the Ss. Except for this the order of presentation of all conditions was randomized for each $\mathrm{S}$.

\section{Results $^{2}$}

The Munsell choices for each $\mathrm{S}$ for each ST over the eight levels of IFs were rank-ordered, giving a low rank to a high Munsell choice. The ranks were summed over the Ss for each level of IF and the mean ranks are given in Table 1 .

Under Condition LW-H the IFs were lighter and more extensively lighter than under any other conditions. Correspondingly, under Condition $\mathrm{RB}-\mathrm{D}$ the IFs were darker and more extensively darker than under any other condition. Analogous to what would be expected were the situation one of simultaneous contrast, these conditions yielded the lowest (1.67) and highest (8.0) mean ranks, respectively (see last row of Table 1 ).

The eight conditions are arranged in Table 1 , reading from left to right, in an approximate order of decreasing lightness of the inducing fields. Under condition $\mathrm{LW}-\mathrm{H}$ the achromatic patches lying near the CO chips were more uniformly lighter than under Conditions RW-M and LW-M. Correspondingly, the CO choices were somewhat higher for Condition $\mathrm{LW}-\mathrm{H}$ than for Conditions RW-M and LW-M (mean ranks of 1.67 vs. 2.33 and 2.5 , respectively). When the achromatic patches near the CO were darkened still further as in Condition RW-D, the Munsell CO choice decreased even further to a mean rank of 3.5. Introducing a black IF adjacent to the STs, even though the subsequent IF varies from light to dark (Conditions LB-M and RB-M) or was mostly light (Condition LB-H) produced still lower Munsell choices and mean ranks of 5.3, 6.7, and 5.8, respectively. It would seem, therefore, that the change in the mean ranks was closely correlated with changes in the lightness and areal size of the interpolated IFs.

It might seem from the data that a white IF at some distance from the $\mathrm{CO}$ (as in Condition $\mathrm{RW}-\mathrm{D}$ ) had a greater effect on the CO than did equally light IFs 
closer to the CO (as in Conditions LB-M, RB-M, LB-H). It should be recalled, however, that the IFs near the ST were lighter and were regarded longer than the IFs near the CO. This lack of symmetry in the IFs and in the duration of their effects could account for the failure of the IFs nearer the CO to produce a greater effect than did the IFs farther from the $\mathrm{CO}$.

The mean ranks at each level of ST (Rows 1-3 in Table 1) support the results described above. At each level of ST, as the lightness of the IFs was decreased, there was a general tendency for the Munsell $\mathrm{CO}$ choices to decrease and therefore for the mean ranks to increase. In comparing the mean ranks for Conditions RW-M and LW-M, as well as for Conditions RB-M and LB-M, it would seem that when all conditions were the same except for the direction of eye-movements, mean ranks were approximately the same: 2.33 and 2.50; 6.67 and 5.30, respectively, although there was a slight tendency for Condition LB-M to produce lower ranks than did Condition RB-M.

A test of Concordance (Kendall's Coefficient W, as reported by Edwards, 1961) for each row of the Table yields W's that individually have $p<.05$ (df $=6 / 103$ ). In comparisons between Conditions $L W-H$ and RB-D, LW-H elicited higher CO choices, 11, 10, and 12 for the 3.5, 5.5, and 7.5 STs, respectively, from the $16 \mathrm{Ss}$, with only 1,3 , and 2 reversals, and 4,3 , and 2 ties.

In a replication of the $\mathrm{LW}-\mathrm{H}$ and $\mathrm{RB}-\mathrm{D}$ conditions with different Es and younger Ss (median age of 16); with larger and therefore numerically fewer trapezoids (but with total area the same); with CO chips measuring $2 \times 2.25$ in., each separated by .25 in., and mounted on a Munsell 2.0 background measuring 14 x 8.5 in.; and with the bottom IFs measuring $11 \times 11$ in., but with all other arrangements the same, LW-H elicited higher CO choices, 12, 11, and 11 from the $16 \mathrm{Ss}$, with only 1,2 , and 2 reversals, respectively. The results of the replication were strikingly like that of the reference experiment. At the end of the replication each $\mathrm{S}$ was instructed to look back and forth at the ST and COs, using the route of the middle black strip, but with all other arrangements the same. With this method of "simultaneous" matching the median Munsell choices (and semi-interquartile ranges) for the three STs were: $3.41(.23), 5.75(.23)$, and $6.90(.35)$, respectively.

\section{Discussion}

In order to explain the pattern of data in Table 1 as being due to the darkening of subsequently-viewed lower reflectance-fields by previously-viewed higher reflec-. tance fields (analogous or even equivalent to the phenomena of successive contrast), one must hypothesize that the inducing effects are predominantly acting forward on the $\mathrm{CO}$ and are not acting backward on the ST. One finding in the data, however, that is disturbing to any explanation in these terms is the failure of the light IFs near the CO to have a greater effect than they did. As explained earlier the first and second IFs were not balanced and that could account for this finding. An experiment is being designed to test this point. There might be a simpler explanation of the data: the $\mathrm{CO}$ choices invariably tend towards an average of the IFs through which the gaze has been routed.

Regardless of the mechanism that one invokes to explain these data, they raise difficulties for the interpretation of experiments and the validation of theories in which the temporal pattern of looking at a compound or complex field or scene has been ignored. Consider, for example, conditions LW-H and RB-D (or RW-M and LB-M; or LW-M and RB-M). The same complex scene was presented to the Ss under both conditions. Nevertheless, when the Ss directed their gaze in a direction that took it through higher inducing fields rather than lower inducing fields, the colormatching responses shifted in a manner seemingly consistent with the degree of lightness of the inducing fields through which the gaze was routed.

\section{References}

Diamond, A. L. Foveal simultaneous brightness contrast as a function of inducing- and test-field luminances. J. exp. Psychol., $1953,45,304-314$

Edwards, A. L. Statistical methods for the behavioral sciences. New York: Holt, Rinehart \& Winston, 1961.

Heinemann, E. G. Simultaneous brightness induction as a function of inducing- and test-field luminances. J. exp. Psychol., 1955, $50,89-96$.

Helson, H. Adaptation-level theory. New York: Harper, 1964. Jameson, Dorothea, \& Hurvich, L. M. Perceived color and its dependence on focal, surrounding and preceding stimulus variables. J. Opt. Soc. Amer., 1959, 49, 890-898.

Judd, D. B. The definition of black and white. Amer. J. Psychol., $1941,54,289-294$.

Leibowitz, H., Mote, F. A., \& Thurlow, W. R. Simultaneous contrast as a function of separation between test and inducing fields. J. exp. Psychol., 1953, 46, 453-456.

\section{Notes}

1. This research was supported by the National Research Council of Canada Grant No. APA-143. The experiment described below was conceived jointly by the senior author and Julian Hochberg during a discussion of successive lightness-judgments, but the authors are solely responsible for its actual design and execution.

2. The tables of raw data for the experiments described above may be obtained free of charge from Psychonomic Press, Box 38, Goleta, Calif., 93017. Document No. PPI-11-3.

(Accepted for publication. September 14, 1966.) 